\title{
Flexure of thick orthotropic plates by exponential shear deformation theory
}

\section{Abstract}

In the present paper, a variationally consistent exponential shear deformation theory taking into account transverse shear deformation effect is presented for the flexural analysis of thick orthotropic plates. The inplane displacement field uses exponential function in terms of thickness coordinate to include the shear deformation effect. The transverse shear stress can be obtained directly from the constitutive relations satisfying the shear stress free surface conditions on the top and bottom surfaces of the plate, hence the theory does not require shear correction factor. Governing equations and boundary conditions of the theory are obtained using the principle of virtual work. Results obtained for static flexure of simply supported orthotropic plates are compared with those of other refined theories and elasticity solution wherever applicable. The results obtained by present theory are in excellent agreement with those of exact results and other higher order theories. Thus the efficacy of the present refined theory is established.

\section{Keywords}

exponential shear deformation theory, static flexure, orthotropic

plates, shear deformation, displacements, transverse shear tresses

\author{
A. S. Sayyad ${ }^{*}$ \\ Department of Civil Engineering, SRES'S \\ College of Engineering, Kopargaon- \\ 423601, (M.S.), India
}

Received 12 Dec 2011

In revised form 13 Aug 2012

*Author email: attu_sayyad@yahoo.co.in

\section{INTRODUCTION}

Classical plate theory $[1,2]$ underestimates the deflection and overestimates natural frequencies and buckling loads. This is due to not taking into account the effect of transverse shear and transverse normal stresses. The errors in deflection and stresses are quite significant for plate made out of advanced composites.

First order shear deformation theory (FSDT) is considered as an improvement over the classical plate theory. Reissner [3, 4] was the first to provide consistent stress-based plate theory, which incorporates the effect of shear deformation; whereas Mindlin [5] developed displacement based first order shear deformation theory. In these theories, the transverse shear strain distribution is assumed to be constant through the plate thickness and therefore, shear correction factor is required to account for the strain energy due to shear deformation. In general, these shear correction factors are problem dependent. 
The limitations of classical and first order shear deformation theories stimulated the development of higher order shear deformation theories, to include effect of cross sectional warping and to get the realistic variation of the transverse shear strains and stresses through the thickness of plate. For higher order theories, primarily two types of approaches are available. In one approach, the stresses are treated as primary variables. In the other approach, displacements are treated as primary variables. Reddy [6] has developed well known higher order shear deformation theory for the analysis of laminated plates considering polynomial function in-terms of thickness co-ordinate to include effect of transverse shear deformation. Many review papers are available on displacement based plate theories [7-9]. Recently many new refined theories [10-12] are developed for the analysis of isotropic and orthotropic plates. Ghugal and Sayyad [13] have developed trigonometric shear deformation theory considering effect of transverse shear and transverse normal strain/stress for the free vibration analysis of thick orthotropic plates. Shimpi and Patel [14] have developed a two variable refined plate theory for orthotropic plate analysis. Thai and Kim [15] applied two variable plate theory for the buckling analysis of orthotropic plates using Levy type solution. Ghugal and Pawar [16] have carried out static flexure analysis of isotropic and orthotropic plates by hyperbolic shear deformation theory. Karama et al. [17] has used exponential function to predict the mechanical behavior multilayered laminated composite beams.

Pagano [18] has developed exact solutions within the framework of the linear theory of elasticity for rectangular bidirectional composites and sandwich plates with pinned edges. The solutions are compared to the respective solutions governed by classical laminated plate theory. However, the nature of exact solution is governed by the specific parameter which depends on the combination of the material, geometric and loading properties.

In the present work, an exponential shear deformation theory is presented for orthotropic plate analysis. The displacement model contains exponential terms in addition to classical plate theory terms. The numbers of unknown variables are same as that of first order shear deformation theory. Exact solution developed by Pagano [18] used as a basis for the comparison of results and to validate the present theory.

\subsection{Orthotropic Plate under Consideration}

Consider a plate (of length $a$, width $b$, and thickness $h$ ) made up of homogenous material. The plate occupies (in $\mathrm{O}-x-y-z$ right-handed Cartesian coordinate system) a region:

$$
0 \leq x \leq a ; \quad 0 \leq y \leq b ; \quad-h / 2 \leq z \leq h / 2
$$

\subsection{Assumptions made in the theory:}

1. The inplane displacement $u$ in $x$ direction as well as displacement $v$ in $y$ direction each consists of two parts:

a) Displacement component analogous to the displacement in classical plate theory of bending.

b) Displacement component due to shear deformation, which is assumed to be exponential in nature with respect to thickness coordinate, such that the maximum shear stress occurs at neutral axis.

Latin American Journal of Solids and Structures 10(2013) $473-490$ 
2. The transverse displacement $w$ in $z$ direction is assumed to be a function of $x$ and y coordinates only.

3. The body forces are ignored in the analysis.

4. The plate can be subjected to transverse loads only.

Based upon the before mentioned assumptions, the displacement field of the present theory is given as below:

$$
\begin{aligned}
& u(x, y, z)=-z \frac{\partial w}{\partial x}+z \exp \left[-2\left(\frac{z}{h}\right)^{2}\right] \phi(x, y) \\
& v(x, y, z)=-z \frac{\partial w}{\partial y}+z \exp \left[-2\left(\frac{z}{h}\right)^{2}\right] \psi(x, y) \\
& w(x, y, z)=w(x, y)
\end{aligned}
$$

Here $u, v$ and $w$ are the displacements in the $x, y$ and $z$ directions. The exponential function in terms of thickness coordinate in both the inplane displacements is associated with the transverse shear stress distribution through the thickness of plate and the functions $\phi$ and $\psi$ are the unknown functions associated with the shear slopes. The normal strains $\varepsilon_{x}, \varepsilon_{y}$ and shear strains $\gamma_{x y}, \gamma_{y z}, \gamma_{z x}$ corresponding to assumed displacement field Eqns. (2) are

$$
\begin{aligned}
& \varepsilon_{x}=\frac{\partial u}{\partial x} \\
& \varepsilon_{y}=\frac{\partial v}{\partial y} \\
& \gamma_{x y}=\frac{\partial u}{\partial x}+\frac{\partial v}{\partial y} \\
& \gamma_{z x}=\frac{\partial u}{\partial z}+\frac{\partial w}{\partial x} \\
& \gamma_{y z}=\frac{\partial v}{\partial z}+\frac{\partial w}{\partial y}
\end{aligned}
$$

Using Eqns. (2) and (3) the normal strains and shear strains are 


$$
\begin{aligned}
& \varepsilon_{x}=-z \frac{\partial^{2} w}{\partial x^{2}}+f(z) \frac{\partial \phi}{\partial x} \\
& \varepsilon_{y}=-z \frac{\partial^{2} w}{\partial y^{2}}+f(z) \frac{\partial \psi}{\partial y} \\
& \gamma_{x y}=-2 z \frac{\partial^{2} w}{\partial x \partial y}+f(z)\left(\frac{\partial \phi}{\partial y}+\frac{\partial \psi}{\partial x}\right) \\
& \gamma_{z x}=\frac{d f(z)}{d z} \phi \\
& \gamma_{y z}=\frac{d f(z)}{d z} \psi
\end{aligned}
$$

where, $f(z)=z \exp \left[-2\left(\frac{z}{h}\right)^{2}\right]$

The constitutive relations for orthotropic materials are as follows

$$
\begin{aligned}
& \sigma_{x}=\bar{Q}_{11} \varepsilon_{x}+\bar{Q}_{12} \varepsilon_{y} ; \\
& \sigma_{y}=\bar{Q}_{12} \varepsilon_{x}+\bar{Q}_{22} \varepsilon_{y} ; \\
& \tau_{x y}=\bar{Q}_{66} \gamma_{x y} ; \\
& \tau_{y z}=\bar{Q}_{44} \gamma_{y z} ; \\
& \tau_{z x}=\bar{Q}_{55} \gamma_{z x}
\end{aligned}
$$

Substituting Eqn. (4) into Eqn. (5)

$$
\begin{aligned}
& \sigma_{x}=\bar{Q}_{11}\left[-z \frac{\partial^{2} w}{\partial x^{2}}+f(z) \frac{\partial \phi}{\partial x}\right]+\bar{Q}_{12}\left[-z \frac{\partial^{2} w}{\partial y^{2}}+f(z) \frac{\partial \psi}{\partial y}\right] \\
& \sigma_{y}=\bar{Q}_{12}\left[-z \frac{\partial^{2} w}{\partial x^{2}}+f(z) \frac{\partial \phi}{\partial x}\right]+\bar{Q}_{22}\left[-z \frac{\partial^{2} w}{\partial y^{2}}+f(z) \frac{\partial \psi}{\partial y}\right] \\
& \tau_{x y}=\bar{Q}_{66}\left[-2 z \frac{\partial^{2} w}{\partial x \partial y}+f(z)\left(\frac{\partial \phi}{\partial y}+\frac{\partial \psi}{\partial x}\right)\right] \\
& \tau_{y z}=\bar{Q}_{44}\left[\frac{d f(z)}{d z} \psi\right] \text { and } \tau_{z x}=\bar{Q}_{55}\left[\frac{d f(z)}{d z} \phi\right]
\end{aligned}
$$


where, $\bar{Q}_{i j}$ are the material properties as follows:

$$
\begin{gathered}
\bar{Q}_{11}=\frac{E_{1}}{1-\mu_{12} \mu_{21}} ; \bar{Q}_{12}=\frac{\mu_{12} E_{1}}{1-\mu_{12} \mu_{21}} ; \bar{Q}_{22}=\frac{E_{2}}{1-\mu_{12} \mu_{21}} ; \\
\bar{Q}_{66}=G_{12} ; \bar{Q}_{55}=G_{13} ; \bar{Q}_{44}=G_{23} .
\end{gathered}
$$

\section{DERIVATION OF GOVERNING EQUATIONS AND BOUNDARY CONDITIONS}

The governing equations and boundary conditions are derived using principle of virtual work. Let $d$ be the arbitrary variations

$$
\iiint_{V}(\delta U-\delta W)=0
$$

where

$$
\delta U=\int_{-h / 2}^{h / 2} \int_{0}^{b} \int_{0}^{a}\left(\sigma_{x} \delta \varepsilon_{x}+\sigma_{y} \delta \varepsilon_{y}+\tau_{y z} \delta \gamma_{y z}+\tau_{z x} \delta \gamma_{z x}+\tau_{x y} \delta \gamma_{x y}\right) d x d y d z
$$

and

$$
\delta W=\int_{0}^{b} \int_{0}^{a} q(x, y) \delta w d x d y
$$

Substituting Eqns. (9) and (10) in Eqn. (8)

$$
\begin{gathered}
\int_{-h / 2}^{h / 2} \int_{0}^{b} \int_{0}^{a}\left(\sigma_{x} \delta \varepsilon_{x}+\sigma_{y} \delta \varepsilon_{y}+\tau_{y z} \delta \gamma_{y z}+\tau_{z x} \delta \gamma_{z x}+\tau_{x y} \delta \gamma_{x y}\right) d x d y d z \\
-\int_{0}^{b} \int_{0}^{a} q(x, y) \delta w d x d y=0
\end{gathered}
$$

Substituting in Eqn. (11) the expressions for displacements [Eqn. (2)], Strains [Eqn. (4)] and stresses [Eqn. (6)] and then employing Green's theorem successively, we obtain the coupled EulerLagrange governing equations of the plate and the associated boundary conditions in terms of stress resultants. The governing differential equations in-terms of stress resultants are as follows: 


$$
\begin{gathered}
\frac{\partial^{2} M_{x}}{\partial x^{2}}+2 \frac{\partial^{2} M_{x y}}{\partial x \partial y}+\frac{\partial^{2} M_{y}}{\partial y^{2}}+q=0 \\
\frac{\partial N_{s x}}{\partial x}+\frac{\partial N_{s x y}}{\partial y}-N_{T c x}=0 \\
\frac{\partial N_{s y}}{\partial y}+\frac{\partial N_{s x y}}{\partial x}-N_{T c y}=0
\end{gathered}
$$

The boundary conditions are of the form:

at $x=0$ and $x=a$

$$
\begin{gathered}
V_{x}=0 \text { or } w \text { is prescribed } \\
M_{x}=0 \text { or } \frac{\partial w}{\partial x} \text { is prescribed } \\
N_{s x}=0 \text { or } \phi \text { is prescribed } \\
N_{s x y}=0 \text { or } \psi \text { is prescribed }
\end{gathered}
$$

at $y=0$ and $y=b$ :

$$
\begin{gathered}
V_{y}=0 \text { or } w \text { is prescribed } \\
M_{y}=0 \text { or } \frac{\partial w}{\partial y} \text { is prescribed } \\
N_{s x y}=0 \text { or } \phi \text { is prescribed } \\
N_{s y}=0 \text { or } \psi \text { is prescribed }
\end{gathered}
$$

at $x(0,0), x(0, a), y(0,0), x(0, b)$

$$
M_{x y}=0 \text { or } w \text { is prescribed }
$$

The stress resultants in the above governing equations and boundary conditions are given as: 


$$
\begin{array}{ll}
M_{x}=\int_{-h / 2}^{h / 2}\left(\sigma_{x} z\right) d z ; & M_{y}=\int_{-h / 2}^{h / 2}\left(\sigma_{y} z\right) d z ; \\
M_{x y}=\int_{-h / 2}^{h / 2}\left(\tau_{x y} z\right) d z ; & N_{s x}=\int_{-h / 2}^{h / 2}\left[\sigma_{x} f(z)\right] d z ; \\
N_{s y}=\int_{-h / 2}^{h / 2}\left[\sigma_{y} f(z)\right] d z ; & N_{s x y}=\int_{-h / 2}^{h / 2}\left[\tau_{x y} f(z)\right] d z ; \\
N_{T c x}=\int_{-h / 2}^{h / 2}\left[\tau_{z x} \frac{d f(z)}{d z}\right] d z ; & N_{T c y}=\int_{-h / 2}^{h / 2}\left[\tau_{y z} \frac{d f(z)}{d z}\right] d z ; \\
V_{x}=\frac{\partial M_{x}}{\partial x}+2 \frac{\partial M_{x y}}{\partial y} ; & V_{y}=\frac{\partial M_{y}}{\partial y}+2 \frac{\partial M_{x y}}{\partial x}
\end{array}
$$

The stress resultants in the Eqn. (24) can be expressed in-terms of functions involved in displacement field by Eqn. (2)

$$
\begin{gathered}
M_{x}=-D_{11} \frac{\partial^{2} w}{d x^{2}}+B s_{11} \frac{\partial \phi}{d x}-D_{12} \frac{\partial^{2} w}{d y^{2}}+B s_{12} \frac{\partial \psi}{d y} \\
M_{y}=-D_{12} \frac{\partial^{2} w}{d x^{2}}+B s_{12} \frac{\partial \phi}{d x}-D_{22} \frac{\partial^{2} w}{d y^{2}}+B s_{22} \frac{\partial \psi}{d y} \\
M_{x y}=-2 D_{66} \frac{\partial^{2} w}{d x \partial y}+B s_{66}\left(\frac{\partial \phi}{d y}+\frac{\partial \psi}{d x}\right) \\
N_{s x}=-B s_{11} \frac{\partial^{2} w}{d x^{2}}+A s s_{11} \frac{\partial \phi}{d x}-B s_{12} \frac{\partial^{2} w}{d y^{2}}+A s s_{12} \frac{\partial \psi}{d y} \\
N_{s y}=-B s_{12} \frac{\partial^{2} w}{d x^{2}}+A s s_{12} \frac{\partial \phi}{d x}-B s_{22} \frac{\partial^{2} w}{d y^{2}}+A s s_{22} \frac{\partial \psi}{d y} \\
N_{s x y}=-2 B s_{66} \frac{\partial^{2} w}{d x \partial y}+A s s_{66}\left(\frac{\partial \phi}{d y}+\frac{\partial \psi}{d x}\right)
\end{gathered}
$$




$$
\begin{gathered}
N_{T c x}=A c c_{55} \phi \\
N_{T c y}=A c c_{44} \psi
\end{gathered}
$$

Using Eqns. (12) through (32) the governing equations and associated boundary conditions of the plate in-terms of functions involved in displacement field are as follows:

$$
\begin{gathered}
D_{11} \frac{\partial^{4} w}{\partial x^{4}}+\left(2 D_{12}+4 D_{66}\right) \frac{\partial^{4} w}{\partial x^{2} \partial y^{2}}+D_{22} \frac{\partial^{4} w}{\partial y^{4}}-B s_{11} \frac{\partial^{3} \phi}{\partial x^{3}}-\left(B s_{12}+2 B s_{66}\right) \frac{\partial^{3} \phi}{\partial x \partial y^{2}} \\
-B s_{22} \frac{\partial^{3} \psi}{\partial y^{3}}-\left(B s_{12}+2 B s_{66}\right) \frac{\partial^{3} \psi}{\partial x^{2} \partial y}=q \\
B s_{11} \frac{\partial^{3} w}{\partial x^{3}}+\left(B s_{12}+2 B s_{66}\right) \frac{\partial^{3} w}{\partial x \partial y^{2}}-A s s_{11} \frac{\partial^{2} \phi}{\partial x^{2}}-A s s_{66} \frac{\partial^{2} \phi}{\partial y^{2}}+A c c_{55} \phi \\
-\left(A s s_{12}+A s s_{66}\right) \frac{\partial^{2} \psi}{\partial x \partial y}=0 \\
B s_{22} \frac{\partial^{3} w}{\partial y^{3}}+\left(B s_{12}+2 B s_{66}\right) \frac{\partial^{3} w}{\partial x^{2} \partial y}-A s s_{66} \frac{\partial^{2} \psi}{\partial x^{2}}-A s s_{22} \frac{\partial^{2} \psi}{\partial y^{2}}+A c c_{44} \psi \\
-\left(A s s_{12}+A s s_{66}\right) \frac{\partial^{2} \phi}{\partial x \partial y}=0
\end{gathered}
$$

The associated consistent boundary conditions at the edges $x=0$ and $x=a$

$$
\begin{gathered}
-D_{11} \frac{\partial^{3} w}{\partial x^{3}}-\left(D_{12}+4 D_{66}\right) \frac{\partial^{3} w}{\partial x \partial y^{2}}+\left(B s_{11} \frac{\partial^{2} \phi}{\partial x^{2}}+2 B s_{66} \frac{\partial^{2} \phi}{\partial y^{2}}\right)+\left(B s_{12}+2 B s_{66}\right) \frac{\partial^{2} \psi}{\partial x \partial y}=0 \\
\text { or } w \text { is prescribed } \\
-D_{11} \frac{\partial^{2} w}{d x^{2}}+B s_{11} \frac{\partial \phi}{d x}-D_{12} \frac{\partial^{2} w}{d y^{2}}+B s_{12} \frac{\partial \psi}{d y}=0 \text { or } \frac{\partial w}{\partial x} \text { is prescribed } \\
-B s_{11} \frac{\partial^{2} w}{d x^{2}}+A s s_{11} \frac{\partial \phi}{d x}-B s_{12} \frac{\partial^{2} w}{d y^{2}}+A s s_{12} \frac{\partial \psi}{d y}=0 \text { or } \phi \text { is prescribed } \\
-2 B s_{66} \frac{\partial^{2} w}{d x \partial y}+A s s_{66}\left(\frac{\partial \phi}{d y}+\frac{\partial \psi}{d x}\right) \text { or } f \text { is prescribed }
\end{gathered}
$$


The associated consistent boundary conditions at the edges $y=0$ and $y=b$ :

$$
\begin{gathered}
-D_{22} \frac{\partial^{3} w}{\partial y^{3}}-\left(D_{12}+4 D_{66}\right) \frac{\partial^{3} w}{\partial x^{2} \partial y}-\left(B s_{22} \frac{\partial^{2} \psi}{\partial y^{2}}+2 B s_{66} \frac{\partial^{2} \psi}{\partial x^{2}}\right)+\left(B s_{12}+2 B s_{66}\right) \frac{\partial^{2} \phi}{\partial x \partial y}=0 \\
\quad \text { or } w \text { is prescribed }
\end{gathered}
$$

At corners:

$$
-2 D_{66} \frac{\partial^{2} w}{d x \partial y}+B s_{66}\left(\frac{\partial \phi}{d y}+\frac{\partial \psi}{d x}\right) \text { or } w \text { is prescribed }
$$

Various constants appear in governing equations and associated boundary conditions are as given below:

$$
\begin{aligned}
& \left(D_{11}, D_{12}, D_{22}, D_{66}\right)=\left(\bar{Q}_{11}, \bar{Q}_{12}, \bar{Q}_{22}, \bar{Q}_{66}\right) \int_{-h / 2}^{+h / 2} z^{2} d z \\
& \left(B s_{11}, B s_{12}, B s_{22}, B s_{66}\right)=\left(\bar{Q}_{11}, \bar{Q}_{12}, \bar{Q}_{22}, \bar{Q}_{66}\right) \int_{-h / 2}^{+h / 2} z f(z) d z \\
& \left(A s s_{11}, A s s_{12}, A s s_{22}, A s s_{66}\right)=\left(\bar{Q}_{11}, \bar{Q}_{12}, \bar{Q}_{22}, \bar{Q}_{66}\right) \int_{-h / 2}^{+h / 2} f^{2}(z) d z \\
& \left(A c c_{44}, A c c_{55}\right)=\left(\bar{Q}_{44}, \bar{Q}_{55}\right) \int_{-h / 2}^{+h / 2}\left[\frac{d f(z)}{d z}\right]^{2} d z .
\end{aligned}
$$




\section{NUMERICAL EXAMPLES}

A simply supported rectangular plate is subjected to transverse loading $q(x, y)=\sum_{m=1,3 \ldots}^{\infty} \sum_{n=1,3 \ldots}^{\infty} q_{m n} \sin \left(\frac{m \pi x}{a}\right) \sin \left(\frac{n \pi y}{b}\right)$ acting on the top surface (i.e., at $\left.z=-h / 2\right)$, where $q_{m n}$ are the coefficients of Fourier expansion of load given as follows for various loading cases.

$$
\begin{aligned}
& q_{m n}=\frac{16 q_{0}}{m n \pi^{2}} \quad \text { Uniformly distributed load } \\
& q_{m n}=q_{0} \quad \text { Singlesine load }
\end{aligned}
$$

The governing differential equations and the associated boundary conditions for static flexure of plate under consideration can be obtained directly from Eqns. (12) through (23). The following are the boundary conditions of the simply supported orthotropic plates.

$$
\begin{array}{ll}
w=\psi=M_{x}=N_{s x}=0 & \text { at } x=0 \text { and } x=a \\
w=\phi=M_{y}=N_{s y}=0 & \text { at } y=0 \text { and } y=b
\end{array}
$$

The following is the solution form for $w(x, y), \phi(x, y)$ and $\psi(x, y)$ assumed for the above examples, which satisfies governing equations and boundary conditions exactly:

$$
\begin{aligned}
& w(x, y)=\sum_{m=1}^{m=\infty} \sum_{n=1}^{n=\infty} w_{m n} \sin \frac{m \pi x}{a} \sin \frac{n \pi y}{b} ; \\
& \phi(x, y)=\sum_{m=1}^{m=\infty} \sum_{n=1}^{n=\infty} \phi_{m n} \cos \frac{m \pi x}{a} \sin \frac{n \pi y}{b} ; \\
& \psi(x, y)=\sum_{m=1}^{m=\infty} \sum_{n=1}^{n=\infty} \psi_{m n} \sin \frac{m \pi x}{a} \cos \frac{n \pi y}{b}
\end{aligned}
$$

where $w_{m n}, \phi_{m n}$ and $\psi_{m n}$ are the unknown coefficients of the respective Fourier expansions and $m$, $n$ are positive integers. In case of single sine load $m=1$ and $n=1$ in the above solution scheme. Substituting this form of solution and the load $q(x, y)$ into the governing equations yields three algebraic simultaneous equations which can be written in following matrix form.

Latin American Journal of Solids and Structures 10(2013) $473-490$ 


$$
\left[\begin{array}{ccc}
K_{11} & K_{12} & K_{13} \\
K_{12} & K_{22} & K_{23} \\
K_{13} & K_{23} & K_{33}
\end{array}\right]\left\{\begin{array}{l}
w_{m n} \\
\phi_{m n} \\
\psi_{m n}
\end{array}\right\}=\left\{\begin{array}{l}
q_{m n} \\
0 \\
0
\end{array}\right\}
$$

Eqn. (48) can be written in more concise form as follows:

$$
[K]\{\Delta\}=\{q\}
$$

where, elements of matrix $[K]$ and vector $\{\Delta\}$ are given as below:

$$
\begin{aligned}
& K_{11}=D_{11} \frac{m^{4} \pi^{4}}{a^{4}}+\left(2 D_{12}+4 D_{66}\right) \frac{m^{2} n^{2} \pi^{4}}{a^{2} b^{2}}+D_{22} \frac{n^{4} \pi^{4}}{b^{4}} \\
& K_{12}=-B s_{11} \frac{m^{3} \pi^{3}}{a^{3}}-\left(B s_{12}+2 B s_{66}\right) \frac{m n^{2} \pi^{3}}{a b^{2}} \\
& K_{13}=-B s_{22} \frac{n^{3} \pi^{3}}{b^{3}}-\left(B s_{12}+2 B s_{66}\right) \frac{m^{2} n \pi^{3}}{a^{2} b} ; \\
& K_{22}=A s s_{11} \frac{m^{2} \pi^{2}}{a^{2}}+A s s_{66} \frac{n^{2} \pi^{2}}{b^{2}}+A c c_{55} ; \\
& K_{23}=\left(A s s_{12}+A s s_{66}\right) \frac{m n \pi^{2}}{a b} \\
& K_{33}=A s s_{22} \frac{n^{2} \pi^{2}}{b^{2}}+A s s_{66} \frac{m^{2} \pi^{2}}{a^{2}}+A c c_{44} ; \\
& \{\Delta\}=\left\{w_{m n} \quad \phi_{m n} \quad \psi_{m n}\right\}^{T}
\end{aligned}
$$

Unknown coefficients $w_{m n}, \phi_{m n}$ and $\psi_{m n}$ can be easily evaluated using Cramer's rule.

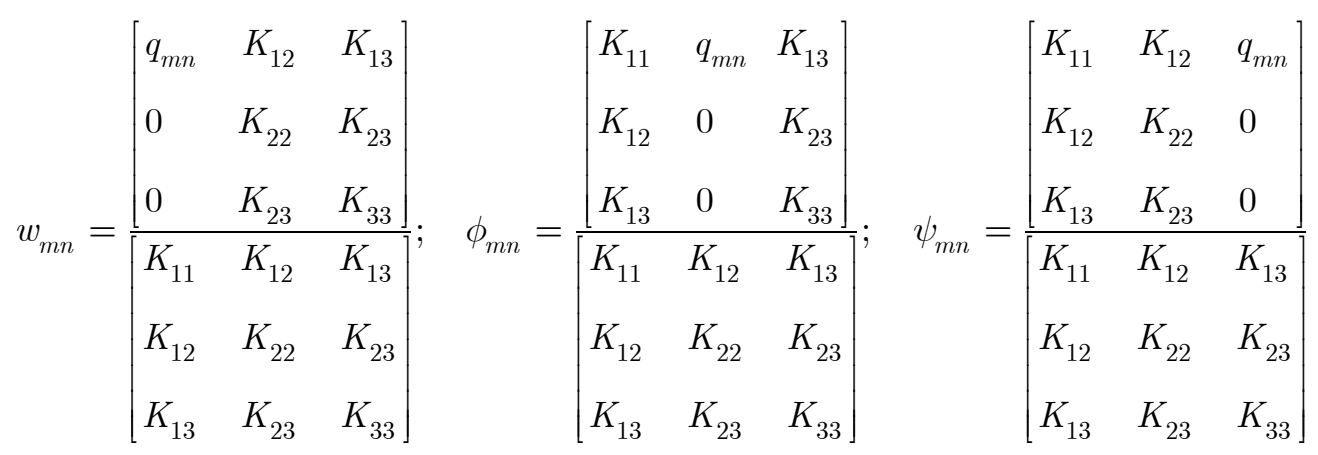


Having obtained values of $w(x, y), \phi(x, y)$ and $\psi(x, y)$ from Eqn. (49) one can then calculate all the displacement and stress components within the plate.

Displacements: Substituting the final solution for $w(x, y), \phi(x, y)$, and $\psi(x, y)$ in the displacement field to obtained the inplane and transverse displacements. The expressions for these displacements are as follows:

$$
\begin{aligned}
& u=\left\{-z \frac{m \pi}{a} w_{m n}+z \exp \left[-2\left(\frac{z}{h}\right)^{2}\right] \phi_{m n}\right\} \cos \frac{m \pi x}{a} \sin \frac{n \pi y}{b} \\
& v=\left\{-z \frac{n \pi}{b} w_{m n}+z \exp \left[-2\left(\frac{z}{h}\right)^{2}\right] \psi_{m n}\right\} \sin \frac{m \pi x}{a} \cos \frac{n \pi y}{b} \\
& w=w_{m n} \sin \frac{m \pi x}{a} \sin \frac{n \pi y}{b}
\end{aligned}
$$

Inplane Stresses: The inplane normal and shear stresses $\left(s_{x}, s_{y}\right.$, and $\left.t_{x y}\right)$ can be obtained by using stress-strain relations as given by the Eqns. (5) and (6).

$$
\begin{aligned}
& \sigma_{x}=\left\{z\left(\bar{Q}_{11} \frac{m^{2} \pi^{2}}{a^{2}}+\bar{Q}_{12} \frac{n^{2} \pi^{2}}{b^{2}}\right) w_{m n}-f(z)\left(\bar{Q}_{11} \frac{m \pi}{a}+\bar{Q}_{12} \frac{n \pi}{b}\right) \phi_{m n}\right\} \sin \frac{m \pi x}{a} \sin \frac{n \pi y}{b} \\
& \left.\sigma_{y}=\left\{z\left(\bar{Q}_{12} \frac{m^{2} \pi^{2}}{a^{2}}+\bar{Q}_{22} \frac{n^{2} \pi^{2}}{b^{2}}\right) w_{m n}-f(z)\left(\bar{Q}_{12} \frac{m \pi}{a}+\bar{Q}_{22} \frac{n \pi}{b}\right) \psi_{m n}\right\} \sin \frac{m \pi x}{a} \sin \frac{n \pi y}{b}\right\} \\
& \tau_{x y}=\bar{Q}_{66}\left[-2 z \frac{m n \pi^{2}}{a b} w_{m n}+f(z)\left(\frac{n \pi}{b} \phi_{m n}+\frac{m \pi}{a} \psi_{m n}\right)\right] \cos \frac{m \pi x}{a} \cos \frac{n \pi y}{b}
\end{aligned}
$$

Transverse Shear Stresses: The transverse shear stresses $\tau_{z x}$ and $\tau_{y z}$ can be obtained either by using the constitutive relations or by integrating equilibrium equations with respect to the thickness coordinate. The expressions for these stresses using constitutive relations are as follows:

$$
\left.\begin{array}{l}
\tau_{z x}=\bar{Q}_{55}\left[\frac{d f(z)}{d z} \phi_{m n}\right] \cos \frac{m \pi x}{a} \sin \frac{n \pi y}{b} \\
\tau_{y z}=\bar{Q}_{44}\left[\frac{d f(z)}{d z} \psi_{m n}\right] \sin \frac{m \pi x}{a} \cos \frac{n \pi y}{b}
\end{array}\right\}
$$

Equilibrium equations of three-dimensional elasticity, ignoring body forces, can be used to obtain transverse shear stresses. These equations are: 


$$
\begin{aligned}
& \frac{\partial \sigma_{x}}{\partial x}+\frac{\partial \tau_{x y}}{\partial y}+\frac{\partial \tau_{z x}}{\partial z}=0 \\
& \frac{\partial \tau_{x y}}{\partial x}+\frac{\partial \sigma_{y}}{\partial y}+\frac{\partial \tau_{y z}}{\partial z}=0
\end{aligned}
$$

Integrating Eqns. (57) and (58) both w.r.t the thickness coordinate $z$ and imposing the following boundary conditions at top and bottom surfaces of the plate

$$
\left[\tau_{z x}\right]_{z= \pm h / 2}=0,\left[\tau_{y z}\right]_{z= \pm h / 2}=0
$$

Expressions for $\tau_{z x}$ and $\tau_{y z}$ can be obtained satisfying the requirements of zero shear stress conditions on the top and bottom surfaces of the plate. The following material properties are used for orthotropic plate given by Pagano [18].

$$
\frac{E_{1}}{E_{2}}=25, \frac{G_{12}}{E_{2}}=\frac{G_{13}}{E_{2}}=0.5, \frac{G_{23}}{E_{2}}=0.2 \text { and } \mu_{12}=\mu_{13}=\mu_{23}=0.25
$$

\section{NUMERICAL RESULTS AND DISCUSSION}

The displacements and stresses are obtained for different aspect ratios $(S=a / h)$ of the plate. The results of exact elasticity theory [18] available in the literature are used as a basis for comparison of results obtained by various plate theories. The results obtained for displacements and stresses are presented in the following non-dimensional parameters.

$$
\bar{w}=\frac{w 100 h^{3} E_{2}}{q a^{4}} ; \quad\left(\bar{\sigma}_{x}, \bar{\sigma}_{y}\right)=\frac{\sigma_{x}, \sigma_{y}}{q S^{2}} ; \quad\left(\bar{\tau}_{z x}, \bar{\tau}_{y z}\right)=\frac{\left(\tau_{z x}, \tau_{y z}\right)}{q S}
$$

$$
\% \text { error }=\frac{\text { value by a particular model }- \text { value by exact elasticity solution }}{\text { value by exact elasticity solution }} \times 100
$$


Table 1 Comparison of inplane displacement $\bar{u}$ at $(x=0, y=b / 2, z=0)$, deflection $\bar{w}$ at $(x=a / 2, y=b / 2, z=0)$, normal stresses $\bar{S}_{x}$ at $(x=a / 2, y=b / 2, z= \pm h / 2)$, normal stresses $\bar{S}_{y}$ at $(x=a / 2, y=b / 2, z= \pm h / 2)$, and $\bar{t}_{x y}$ at $(x=0, y$ $=b / 2, z= \pm h / 2)$ in orthotropic square plate subjected to sinusoidal load

\begin{tabular}{llllllll}
\hline \multirow{2}{*}{$S$} & Theory & Model & $\bar{u}$ & $\bar{w}$ & $\bar{\sigma}_{\boldsymbol{x}}$ & $\bar{\sigma}_{\boldsymbol{y}}$ & $\bar{\tau}_{x y}$ \\
\hline \multirow{4}{*}{4} & Present & ESDT & 0.0094 & 1.5828 & 0.7765 & 0.0667 & 0.0312 \\
& Reddy [6] & HSDT & 0.0092 & 1.6206 & 0.7379 & 0.0640 & 0.0289 \\
& Mindlin [5] & FSDT & 0.0060 & 1.6616 & 0.4784 & 0.0579 & 0.0358 \\
& Kirchhoff [1, 2] & CPT & 0.0068 & 0.431 & 0.5387 & 0.0267 & 0.0213 \\
& Pagano [18] & Elasticity & 0.0093 & 1.5978 & 0.7276 & 0.0727 & -- \\
\hline \multirow{2}{*}{10} & Present & ESDT & 0.0071 & 0.6327 & 0.5809 & 0.0348 & 0.0229 \\
& Reddy [6] & HSDT & 0.0071 & 0.6371 & 0.5700 & 0.0347 & 0.0225 \\
& Mindlin [5] & FSDT & 0.0066 & 0.6383 & 0.5385 & 0.0339 & 0.0246 \\
& Kirchhoff [1,2] & CPT & 0.0068 & 0.431 & 0.5387 & 0.0267 & 0.0213 \\
& Pagano [18] & Elasticity & 0.0071 & 0.634 & 0.5680 & 0.0360 & --- \\
\hline \multirow{2}{*}{100} & Present & ESDT & 0.0068 & 0.4333 & 0.5391 & 0.0268 & 0.0213 \\
Reddy [6] & Mindlin [5] & HSDT & 0.0068 & 0.4333 & 0.5390 & 0.0268 & 0.0213 \\
& Kirchhoff [1, 2] & FSDT & 0.0068 & 0.4333 & 0.5385 & 0.0267 & 0.0213 \\
& Pagano [18] & CPT & 0.0068 & 0.431 & 0.5387 & 0.0267 & 0.0213 \\
\hline
\end{tabular}

Table 2 Comparison of transverse shear stress $\bar{\tau}_{z x}$ at $(x=0, y=b / 2, z=0)$ and transverse shear stress $\bar{\tau}_{z y}$ at $(x=a / 2, y=0$, $z=0)$ in orthotropic square plate subjected to sinusoidal load

\begin{tabular}{|c|c|c|c|c|c|c|}
\hline$S$ & Theory & Model & $\bar{\tau}_{x z}^{C R}$ & $\bar{\tau}_{x z}^{E E}$ & $\bar{\tau}_{y z}^{C R}$ & $\bar{\tau}_{y z}^{E E}$ \\
\hline \multirow{5}{*}{4} & Present & ESDT & 0.3938 & 0.3764 & 0.0717 & 0.0697 \\
\hline & Reddy [6] & HSDT & 0.3903 & 0.3532 & 0.0714 & 0.0694 \\
\hline & Mindlin [5] & FSDT & 0.2692 & 0.4039 & 0.0491 & 0.0736 \\
\hline & Kirchhoff $[1,2]$ & $\mathrm{CPT}$ & -- & 0.4397 & --- & 0.0377 \\
\hline & Pagano [18] & Elasticity & 0.3620 & --- & 0.0738 & --- \\
\hline \multirow{5}{*}{10} & Present & ESDT & 0.4375 & 0.4265 & 0.0466 & 0.0455 \\
\hline & Reddy [6] & HSDT & 0.4290 & 0.4225 & 0.0458 & 0.0455 \\
\hline & Mindlin [5] & FSDT & 0.2877 & 0.4315 & 0.0306 & 0.0459 \\
\hline & Kirchhoff $[1,2]$ & $\mathrm{CPT}$ & --- & 0.4397 & --- & 0.0377 \\
\hline & Pagano [18] & Elasticity & 0.422 & --- & 0.0460 & --- \\
\hline \multirow{5}{*}{100} & Present & ESDT & 0.4493 & 0.4397 & 0.0386 & 0.0377 \\
\hline & Reddy [6] & HSDT & 0.4397 & 0.4396 & 0.0378 & 0.0377 \\
\hline & Mindlin [5] & FSDT & 0.2931 & 0.4397 & 0.0252 & 0.0377 \\
\hline & Kirchhoff $[1,2]$ & $\mathrm{CPT}$ & --- & 0.4397 & --- & 0.0377 \\
\hline & Pagano [18] & Elasticity & 0.4390 & --- & 0.0377 & --- \\
\hline
\end{tabular}

Latin American Journal of Solids and Structures 10(2013) $473-490$ 
Table 3 Comparison of inplane displacement $\bar{u}$ at $(x=0, y=b / 2, z=0)$, deflection $\bar{w}$ at $(x=a / 2, y=b / 2, z=0)$, normal stresses $\bar{\sigma}_{x}$ at $(x=a / 2, y=b / 2, z= \pm h / 2)$, normal stresses $\bar{\sigma}_{y}$ at $(x=a / 2, y=b / 2, z= \pm h / 2)$, and $\bar{\tau}_{x y}$ at $(x=0, y$ $=b / 2, z= \pm h / 2)$ in orthotropic square plate subjected to uniformly distributed load

\begin{tabular}{|c|c|c|c|c|c|c|c|}
\hline$S$ & Theory & Model & $\bar{u}$ & $\bar{w}$ & $\bar{\sigma}_{x}$ & $\bar{\sigma}_{y}$ & $\bar{\tau}_{x y}$ \\
\hline \multirow{5}{*}{4} & Present & ESDT & 0.0156 & 2.3368 & 1.0754 & 0.0740 & 0.0805 \\
\hline & Reddy [6] & HSDT & 0.0147 & 2.3886 & 1.0188 & 0.0746 & 0.0739 \\
\hline & Mindlin [5] & FSDT & 0.0092 & 2.4375 & 0.7041 & 0.0727 & 0.0742 \\
\hline & Kirchhoff $[1,2]$ & $\mathrm{CPT}$ & 0.0104 & 0.6497 & 0.7867 & 0.0245 & 0.0464 \\
\hline & Pagano [18] & Elasticity & 0.0146 & 2.3590 & 0.9640 & 0.0780 & --- \\
\hline \multirow{5}{*}{10} & Present & ESDT & 0.0113 & 0.9444 & 0.8341 & 0.0353 & 0.0514 \\
\hline & Reddy [6] & HSDT & 0.0111 & 0.9506 & 0.8246 & 0.0355 & 0.0497 \\
\hline & Mindlin [5] & FSDT & 0.0102 & 0.9520 & 0.7707 & 0.0353 & 0.0540 \\
\hline & Kirchhoff $[1,2]$ & $\mathrm{CPT}$ & 0.0104 & 0.6497 & 0.7867 & 0.0245 & 0.0464 \\
\hline & Pagano [18] & Elasticity & 0.0112 & 0.9470 & 0.8210 & 0.0360 & --- \\
\hline \multirow{5}{*}{100} & Present & ESDT & 0.0104 & 0.6528 & 0.7873 & 0.0246 & 0.0447 \\
\hline & Reddy [6] & HSDT & 0.0104 & 0.6528 & 0.7871 & 0.0246 & 0.0445 \\
\hline & Mindlin [5] & FSDT & 0.0104 & 0.6528 & 0.7866 & 0.0246 & 0.0465 \\
\hline & Kirchhoff $[1,2]$ & $\mathrm{CPT}$ & 0.0104 & 0.6497 & 0.7867 & 0.0245 & 0.0464 \\
\hline & Pagano [18] & Elasticity & 0.0104 & 0.6527 & 0.7870 & 0.0240 & --- \\
\hline
\end{tabular}

Table 4 Comparison of transverse shear stress $\bar{\tau}_{z x}$ at $(x=0, y=b / 2, z=0)$ and transverse shear stress $\bar{\tau}_{z y}$ at $(x=a / 2, y=0$, $z=0)$ in orthotropic square plate subjected to uniformly distributed load

\begin{tabular}{|c|c|c|c|c|c|c|}
\hline$S$ & Theory & Model & $\bar{\tau}_{z x}^{C R}$ & $\bar{\tau}_{x z}^{E E}$ & $\bar{\tau}_{y z}^{C R}$ & $\bar{\tau}_{y z}^{E E}$ \\
\hline \multirow{5}{*}{4} & Present & ESDT & 0.6542 & 0.6244 & 0.2172 & 0.2175 \\
\hline & Reddy [6] & HSDT & 0.6567 & 0.6166 & 0.2183 & 0.1885 \\
\hline & Mindlin [5] & FSDT & 0.4906 & 0.7359 & 0.1575 & 0.2362 \\
\hline & Kirchhoff $[1,2]$ & $\mathrm{CPT}$ & --- & 0.7806 & --- & 0.1846 \\
\hline & Pagano [18] & Elasticity & 0.6160 & --- & 0.2060 & --- \\
\hline \multirow{5}{*}{10} & Present & ESDT & 0.7564 & 0.7259 & 0.1935 & 0.1870 \\
\hline & Reddy [6] & HSDT & 0.7469 & 0.6813 & 0.1909 & 0.1810 \\
\hline & Mindlin [5] & FSDT & 0.5154 & 0.7731 & 0.1299 & 0.1949 \\
\hline & Kirchhoff $[1,2]$ & $\mathrm{CPT}$ & --- & 0.7806 & --- & 0.1846 \\
\hline & Pagano [18] & Elasticity & 0.7310 & --- & 0.1880 & --- \\
\hline \multirow{5}{*}{100} & Present & ESDT & 0.7970 & 0.7796 & 0.1887 & 0.1846 \\
\hline & Reddy [6] & HSDT & 0.7800 & 0.7789 & 0.1847 & 0.1846 \\
\hline & Mindlin [5] & FSDT & 0.5203 & 0.7805 & 0.1232 & 0.1847 \\
\hline & Kirchhoff $[1,2]$ & $\mathrm{CPT}$ & --- & 0.7806 & --- & 0.1846 \\
\hline & Pagano [18] & Elasticity & 0.7790 & --- & 0.1841 & --- \\
\hline
\end{tabular}




\subsection{Discussion of Results}

Table 1 shows the comparison of inplane displacement, transverse (central) deflection, inplane normal stresses and inplane shear stress for the orthotropic plate subjected to single sine load for the various aspect ratios. For aspect ratios $(S=a / h) 10$ and 100 present theory and Reddy's [6] theory predicts exact results for inplane displacement whereas theory of Mindlin [5] and Kirchhoff [1, 2] underestimates the same. Central deflection predicted by present theory is in excellent agreement with that of exact solution for all the aspect ratios. Theories of Reddy [6] and Mindlin [5] overestimate the central deflection for aspect ratios 4 and 10. Present theory yields higher value for the inplane normal stress $\left(\bar{\sigma}_{x}\right)$ for all the aspect ratios whereas it is in good agreement when predicted by the theory of Reddy [6]. Inplane normal stress $\left(\bar{\sigma}_{y}\right)$ predicted by present theory is in excellent agreement with exact solution. Reddy [6] underestimates the value of inplane normal stress $\left(\bar{\sigma}_{y}\right)$ for all the aspect ratios. CPT [1, 2] and FSDT [5] yield the lower values for inplane normal stresses for all the aspect ratios.

Comparison of transverse shear stresses for the orthotropic plate subjected to single sine load is presented in Table 2. Examination of Table 2 reveals that, for $S=4$, present theory overpredicts the value of maximum transverse shear stress $\left(\bar{\tau}_{z x}\right)$ by $8.781 \%$ when obtained using constitutive relation $\left(\bar{\tau}_{x z}^{C R}\right)$ and overpredicts the same by $3.98 \%$ when obtained using equilibrium equations $\left(\bar{\tau}_{x z}^{E E}\right)$. For $S=10$, present theory and theory of Reddy [6] shows good accuracy of results. CPT [1, 2] and FSDT [5] overestimate the value of maximum transverse shear stress $\left(\bar{\tau}_{x z}^{E E}\right)$ when obtained via equations of equilibrium. Present theory and theory of Reddy [6] shows good accuracy of results for maximum transverse shear stress $\left(\bar{\tau}_{y z}\right)$ when obtained using constitutive relation.

Comparison of deflection and stresses of an orthotropic plate subjected to uniformly distributed load are shown in Tables 3 and 4. Observation of Table 3 shows that, present theory predicts higher value of inplane displacement for all the aspect ratios. The central deflections predicted by the present theory are in close agreement with those of exact elasticity solution for all the aspect ratios. The percentage error in central deflection is $-0.94 \%,-0.27 \%$ and $0.015 \%$ using present theory for aspect ratios 4, 10 and 100 respectively. The Reddy's theory [6] and Mindlin's theory [5] overpredicts the value of central deflection. The present theory yields the improved inplane normal stresses compared to other theories. The percentage error in inplane normal stress $\left(\bar{\sigma}_{y}\right)$ is $-5.12 \%$ and $1.94 \%$ for $S=4$ and $S=10$ respectively using present theory. Inplane normal stresses predicted by Reddy's theory [6] are in good agreement with those of exact solution. FSDT [5] and CPT [1, 2] yield lower values for inplane displacement and normal stresses. Examination of Table 4 reveals that, the transverse shear stresses predicted by present theory and Reddy's theory [6] are in excellent agreement with those of exact solution when obtained by equilibrium equations.

Latin American Journal of Solids and Structures 10(2013) $473-490$ 


\section{CONCLUSIONS}

In this paper, exponential shear deformation theory is applied to static flexure of square orthotropic plates and following conclusions are drawn from the study.

1. It is variationally consistent displacement based refined shear deformation theory.

2. The constitutive relations are satisfied in respect of inplane stress and transverse shear stress.

3. The transverse shear stresses satisfy shear stress free boundary conditions at the top and bottom faces of the plate hence the theory does not require shear correction factor.

4. It is observed that the results of central deflection predicted by proposed theory is in excellent agreement with the exact results.

5. Inplane stresses predicted by proposed theory are in good agreement with the exact results and other higher order theories.

6. The proposed theory is capable of producing reasonably good transverse shear stresses using constitutive relations, and better values of these stresses can be obtained by integration of equilibrium equations.

\section{References}

[1] G.R. Kirchhoff. Uber das gleichgewicht und die bewegung einer elastischen scheibe. Journal of Reine Angew. Math. (Crelle), 40:51-88, 1850.

[2] G.R. Kirchhoff. Uber die uchwingungen einer kriesformigen elastischen scheibe, Poggendorffs Annalen. 81:58$264,1850$.

[3] E. Reissner. On the theory of bending of elastic plates. Journal of Mathematics and Physics, 23:184-191, 1944.

[4] E. Reissner. The effect of transverse shear deformation on the bending of elastic plates. ASME Journal of Applied Mechanics, 12:69-77, 1945.

[5] R.D. Mindlin. Influence of rotatory inertia and shear on flexural motions of isotropic, elastic plates. ASME Journal of Applied Mechanics, 18:31-38, 1951.

[6] J. N. Reddy. A simple higher order theory for laminated composite plates. ASME Journal of Applied Mechanics, 51:745-752, 1984.

[7] Y.M. Ghugal and R.P. Shimpi. A review of refined shear deformation theories for isotropic and anisotropic laminated plates. Journal of Reinforced Plastics and Composites, 21: 775-813, 2002.

[8] W. Chen and W. Zhen. A selective review on recent development of displacement-based laminated plate theories. Recent Patents on Mechanical Engineering, 1:29-44, 2008.

[9] I. Kreja. A literature review on computational models for laminated composite and sandwich panels. Central European Journal of Engineering, 1(1):59-80, 2011.

[10] R. P. Shimpi. Refined plate theory and its variants. AIAA Journal, 40(1): 137-146, 2002.

[11] R. P. Shimpi, H. G. Patel and H. Arya. New first order shear deformation plate theories. Journal of Applied Mechanics, 74: 523-533, 2007.

[12] Y. M. Ghugal and A. S. Sayyad. A flexure of thick isotropic plate using trigonometric shear deformation theory. Journal of Solid Mechanics, 2(1):79-90, 2010.

[13] Y. M. Ghugal and A. S. Sayyad. Free vibration analysis of thick orthotropic plates using trigonometric shear deformation theory. Latin American Journal of Solids and Structures, 8: 229-243, 2011.

[14] R. P. Shimpi and H. G. Patel. A two variable refined plate theory for orthotropic plate analysis. International Journal of Solids and Structures, 43:6783-6799, 2006.

[15] H. Thai and S. Kim. Levy-type solution for buckling analysis of orthotropic plates based on two variable refined plate theory. Composite Structures, 93: 1738-1746, 2011. 
[16] Y. M. Ghugal and M. D. Pawar. Flexural analysis of thick plates by hyperbolic shear deformation theory. Journal of Experimental \& Applied Mechanics, 2(1):1-21, 2011.

[17] M. Karama, K. S. Afaq and S. Mistou. Mechanical behavior of laminated composite beam by new multi-layered laminated composite structures model with transverse shear stress continuity. International Journal of Solids and Structures, 40:1525-1546, 2003.

[18] N. J. Pagano. Exact solutions for bidirectional composites and sandwich plates. Journal of Composite Material, 4:20-34, 1970.

Latin American Journal of Solids and Structures 10(2013) $473-490$ 